15

\title{
Моделирование процесса теплоотдачи сверхпроводящих пленок в резистивном состоянии
}

\author{
(C) М.А. Васютин, Н.Д. Кузьмичев, Д.А. Шилкин \\ Мордовский государственный университет им. Н.П. Огарёва, \\ 430005 Саранск, Россия \\ e-mail: vasyutinm@mail.ru
}

Поступило в Редакцию 28 мая 2020 г.

В окончательной редакции 12 сентября 2020 г.

Принято к публикации 13 сентября 2020 г.

Проведен анализ теплопереноса в системе контакты-пленка-подложка в условиях, когда отвод тепла от образца в подложку недостаточен для обеспечения отсутствия перегрева образца. Для низких температур предложен метод увеличения теплоотвода от тонкопленочных образцов при пропускании через них электрического тока большой плотности. В качестве основного фактора усиления теплоотвода использовано свойство аномально высокой теплопроводности меди при температурах от 5 до $50 \mathrm{~K}$. Численно решено уравнение теплопроводности для системы пленка-подложка при условии дополнительной теплооддачи в потенциальные контакты. Показано, что контакты из бериллиевой бронзы могут обеспечивать эффективный теплоотвод от образцов сверхпроводящих пленок, находящихся в резистивном состоянии, в условиях сильного джоулевого тепловыделения.

Ключевые слова: вольт-амперные характеристики, сверхпроводящие пленки, теплопроводность, бериллиевая бронза, неоднородное уравнение теплопроводности, 3-я краевая задача.

DOI: $10.21883 /$ JTF.2021.03.50534.183-20

\section{Введение}

При проведении экспериментов по исследованию вольт-амперных характеристик (BAX) и других параметров образцов, связанных с пропусканием через них сильного тока, необходимым является выполнение условия достаточного теплоотвода. Особенно важно это тогда, когда изучаются сверхпроводящие пленки в резистивном состоянии, переходящие в нормальное состояние при перегреве. Условно образцы пленок можно разделить на две группы: нанометрового размера (вискеры, узкие каналы и т.п.) и микрометрового (широкие пленки, ленты и др.). Если образцы первого типа используются обычно в качестве детекторов $[1,2]$, и уменьшение размеров здесь напрямую связано с увеличением чувствительности, то вторая группа часто применяется в сильноточной технике, где и необходимо учитывать нагревание токонесущих элементов. Применение широких и относительно толстых пленок возможно в сверхпроводящих магнитах и накопителях энергии, которые исследуются сейчас в качестве альтернативы аккумуляторам [3-5].

При изучении токонесущих свойств тонких пленок для формирования контактов используют напыление, ультразвуковую сварку и другие методы, сильно влияющие на образец. Его повторное исследование в других экспериментах после этого невозможно. Магнитные измерения лишь косвенно позволяют определять транспортные характеристики [6]. Комплексное изучение сильноточных характеристик образца неразрушающими методами можно обеспечить прижимными контактами. В этом случае материал контактов должен обладать не только хорошей электропроводностью, но и высокими характеристиками упругих свойств. В настоящей работе исследуются контакты из бериллиевой бронзы $(\mathrm{BeCu})$.

\section{1. Физические свойства контактов. Оценка теплоотвода}

Рассмотрим систему контакты-пленка-подложка-медная основа на примере сверхпроводящей пленки нитрида ниобия $(\mathrm{NbN})$, напыленной на кварцевую подложку (рис. 1). Пленка нитрида ниобия обладает высоким удельным сопротивлением в нормальном состоянии. В качестве материала контактов используем бериллиевую бронзу толщиной $0.13 \mathrm{~mm}$. Упругие свойства бериллиевой бронзы, в частности, предел усталости превосходят даже свойства пружинной стали, что вместе с высокой коррозионной стойкостью делает этот материал чрезвычайно подходящим для изготовления пружин и прижимных контактов [7]. Кроме этого, бериллиевая бронза обладает высокой электро- и теплопроводностью (содержит 98\% меди), что особенно важно при проведении транспортных исследований.

Электропроводность бериллиевой бронзы составляет $30 \%$ от электропроводности меди [8], которая при температуре $T=14.5 \mathrm{~K}$ имеет значение $\left.\sigma=7.143 \cdot 10^{9} \mathrm{~S} / \mathrm{m}\right)[8]$. Теплопроводность бериллиевой бронзы определяется формулой Смита и Палмера для сплава [9]:

$$
\lambda=2.392 \cdot 10^{-8} \sigma T+7.539 .
$$




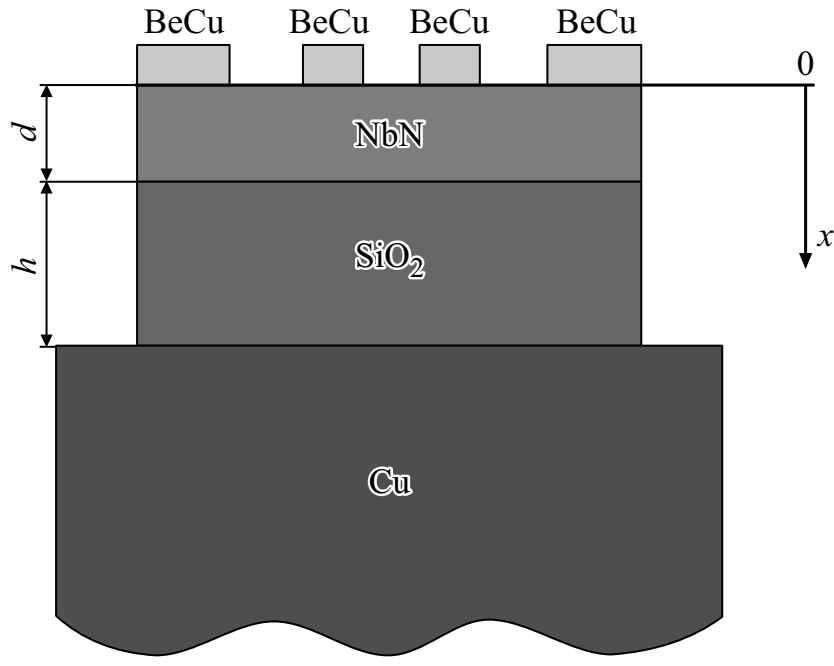

Рис. 1. Схематическое расположение пленки с контактами, подложки и медной основы.

Тогда, подставляя электропроводность бериллиевой бронзы $\sigma_{\mathrm{BeCu}}$ в формулу $(1)$, получим для ее теплопроводности $-\lambda_{\mathrm{BeCu}}=751 \mathrm{~W} /(\mathrm{m} \cdot \mathrm{K})$.

При экспериментальном исследовании ВАХ пленки необходимо обеспечить хороший теплоотвод, чтобы ее нагрев не превышал нескольких десятых градуса. Для оценки повышения температуры $\Delta T$ пленки при пропускании через нее одиночного импульса, представляющего собой линейно увеличивающийся от времени ток, будем считать, что все выделившееся в ней тепло $Q_{1}$ поглощается потенциальными контактами. Масса контактов $\mathrm{mBeCu}$ более чем на два порядка превышает массу пленки (исследуемые образцы имели длину $l=9 \mathrm{~mm}$, ширину $b=5 \mathrm{~mm}$, толщину $d=400 \mathrm{~nm}$, температура перехода пленок в сверхпроводящее состояние $T_{c}$ находилась в пределах $16.2-16.5 \mathrm{~K}$, ширина перехода $\sim 0.1 \mathrm{~K}$.). Тогда $\Delta T=Q_{1} /\left(m_{\mathrm{BeCu}} c_{\mathrm{BeCu}}\right)$, $Q_{1}=(1 / 3) R\left(I_{\max }\right)^{2} t_{0}$. Здесь $R=2.5 \Omega-$ сопротивление пленки в нормальном состоянии, $I_{\max }=1.12 \mathrm{~A}-$ максимальное значение тока, $t_{0}$ - длительность импульса тока, $c_{\mathrm{BeCu}} \approx 7.5 \mathrm{~J} /(\mathrm{kg} \cdot \mathrm{K})$ - удельная теплоемкость $\mathrm{BeCu}$, близкая к теплоемкости меди при $T=20 \mathrm{~K}$ [8], $m_{\mathrm{BeCu}}=40 \mathrm{mg}$. Для $t_{0}=25 \mu \mathrm{s}$ получим $\Delta T=0.09 \mathrm{~K}$.

Оценим отношение мощностей $P_{1} / P_{2}$ тепловыделения $P_{1}=(1 / 3) R\left(I_{\max }\right)^{2}$ и теплопоглощения $P_{2}=$ $=\lambda_{\mathrm{BeCu}}(\partial T / \partial x) S\left(S=0.1 \mathrm{~cm}^{2}\right.$ - площадь потенциальных контактов, составляющая около $20 \%$ площади пленки), $\partial T / \partial x=0.7 \mathrm{~K} / \mathrm{mm}$ - градиент температуры в потенциальных контактах при повышении температуры на $\Delta T$ при толщине контактов $\Delta x=0.13 \mathrm{~mm}$. Тогда $P_{1} / P_{2} \approx 0.2$. Высокая мощность теплопоглощения обусловлена увеличением теплопроводности меди более, чем на порядок при температурах от 5 до $50 \mathrm{~K}[8,9]$.

\section{2. Моделирование процесса теплопереноса}

Учитывая высокую теплопроводность контактов и еще более высокую теплопроводность медной основы, можно ограничиться анализом процесса нагрева пленки и подложки. Температуру контактов и медной основы будем считать постоянной и равной температуре, поддерживаемой в эксперименте.

Считая, что тепло равномерно распределяется по сечениям, параллельным поверхностям пленки и подложки, можно свести задачу к одномерному случаю. При этом изменение температуры будет происходить лишь по оси $x$.

Для нахождения распределения температуры в системе пленка-подложка при пропускании через пленку импульса тока используем неоднородное уравнение теплопроводности:

$$
\frac{\partial T}{\partial t}=a^{2} \frac{\partial^{2} T}{\partial x^{2}}+f(x, t),
$$

где $\quad a^{2}=\lambda / c_{\mathrm{p}} \rho=4.3 \cdot 10^{-2} \mathrm{~cm}^{2} / \mathrm{s} \quad$ - коэффициент температуропроводности подложки, $c_{\mathrm{p}}=17 \mathrm{~J} /(\mathrm{kg} \cdot \mathrm{K})-$ удельная теплоемкость подложки при $20 \mathrm{~K}$ [8], $\rho=2200 \mathrm{~kg} / \mathrm{m}^{3}$ - плотность подложки, $\lambda=$ $=0.16 \mathrm{~W} /(\mathrm{m} \cdot \mathrm{K})-$ теплопроводность кварцевой подложки при $20 \mathrm{~K}, T$ - температура пленки, $t-$ время, $x$ - координата с началом на внешней от подложки плоскости пленки, направленная перпендикулярно в сторону подложки (рис. 1). $f(x, t)=P(x, t) /\left(c_{\mathrm{NbN}} \cdot \rho_{\mathrm{NbN}}\right)$, $P(x, t)$ - объемная плотность мощности источника тепла (пленки), $\quad c_{\mathrm{NbN}}=17 \mathrm{~J} /(\mathrm{kg} \cdot \mathrm{K})-$ удельная теплоемкость пленки при $T=13.5 \mathrm{~K}$ (сумма решеточной и электронной теплоемкости), $\rho_{\mathrm{NbN}}=$ $=8400 \mathrm{~kg} / \mathrm{m}^{3}$ - плотность пленки. Учитывая, что сила тока линейно возрастает за время $t_{0}$ от 0 до $I_{\max }$, величина $P(x, t)$ определяется законом Джоуля-Ленца:

$$
P(x, t)=\rho j_{0}^{2} t^{2}, \quad \text { если } \quad x \leq d \quad \text { и } \quad t \leq t_{0} .
$$

Здесь $\rho \approx 440 \mu \Omega \cdot \mathrm{cm}-$ удельное сопротивление пленки в нормальном состоянии, $j_{0}=I_{\max } /\left(d b t_{0}\right)$.

Уравнение теплопроводности (2) можно решать численно без нахождения явной функции. Начальным условием для нашей задачи будет $T(x, 0)=13.5 \mathrm{~K}$. Левое граничное условие (верхняя граница пленки) $\partial T(0, t) / \partial x=H[T(0, t)-13.5 \mathrm{~K}]$ (3-я краевая задача), правое граничное условие (нижняя граница подложки) - $T(h+d, t)=13.5 \mathrm{~K}$ (1-я краевая задача). Здесь $h$ - толщина подложки $(h \gg d)$, а $H$ - параметр, равный отношению теплоотдачи к теплопроводности материала $\left([H]=m^{-1}\right)$. После неявной разностной аппроксимации уравнения (2) для решения полученной системы линейных уравнений с трехдиагональной матрицей применялся метод прогонки [10].

Параметр $H$ был найден с помощью симметрии задачи. Для этого вначале находилось такое значение $H$, при 
котором максимум нагрева пленки приходился на ее середину $(x=0.2 \mu \mathrm{m})$. В этом случае теплоотдача верхней и нижней поверхностей пленки будет одинаковой. Так как теплопроводности подложки и контактов известны, то можно оценить требуемую $H$, учитывая, что площадь контактов составляет 20\% площади пленки. Было получено $H=50 \mu \mathrm{m}^{-1}$. Для этого значения параметра решения уравнения (2) представлены на рис. 2. Из рисунка видно, что температура пленки быстрее всего нарастает на ее нижнем крае (граничащем с подложкой). После прекращения подачи тока температура быстро спадает в пленке и подложке. Максимальный нагрев пленки имеет значение $\Delta T \approx 0.2 \mathrm{~K}$.

На рис. 3 показана зависимость максимальной температуры локального нагрева $\Delta T_{\max }$ от $H$. Максимальный нагрев пленки мало меняется для $H$, большей $20 \mu \mathrm{m}^{-1}$, не опускаясь ниже $0.17 \mathrm{~K}$ (при приближении к первой краевой задаче). Таким образом, расчетное значение $H$ взято с большим запасом, что доказывает возможность использования применяемого метода экспериментального исследования ВАХ.

Уравнение (2) можно решить и аналитически. Его решение для полубесконечной прямой $(d \ll h)$ с теми же условиями имеет вид

$$
T(x, t)=\int_{0}^{t} \int_{0}^{d} G(x, z, t-\tau) f(z, \tau) d z d \tau,
$$

где $G(x, z, t-\tau)$ - функция Грина для 3-й краевой задачи [11]. Нахождение функции $T(x, t)$ производилось также численно. Полученные зависимости были аналогичны изображенным на рис. 2. При этом максимальное повышение температуры имело значение около $0.2 \mathrm{~K}$, что совпадает с вышеприведенным результатом численного решения уравнения (2).

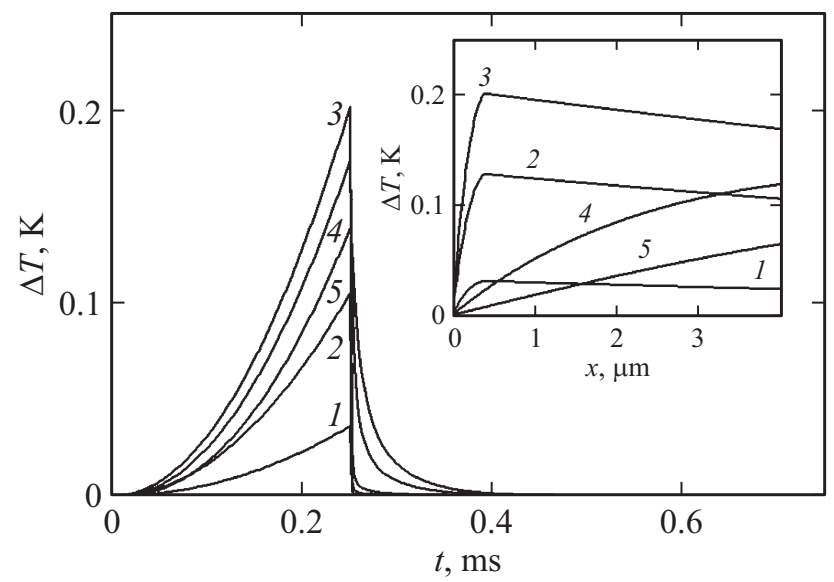

Рис. 2. Результаты расчета нагрева пленки $\mathrm{NbN}$ в зависимости от времени для разных $x, \mu \mathrm{m}: 1-0.01,2-0.1,3-0.4$, $4-3.5,5-8$. На вставке приведены зависимости нагрева пленки от $x$ для разных времен $t, \mu \mathrm{s}: 1-100,2-200,3-$ $250,4-252,5-256$.

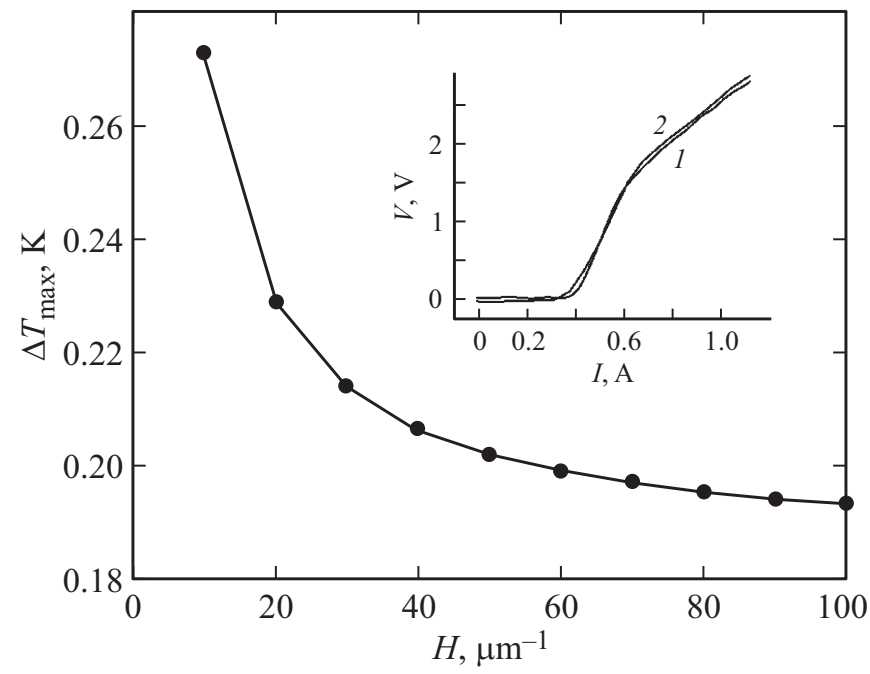

Рис. 3. Зависимость температуры максимального локального нагрева пленки $\mathrm{NbN}$ от параметра $H$. На вставке показаны BAX пленки $\mathrm{NbN}$ при $T=13.5 \mathrm{~K}$ в магнитном поле с индукцией $B=6$ Т для разных $t_{0}(1-250,2-25 \mu \mathrm{s})$.

\section{3. Эксперимент}

BAX сверхпроводящих образцов нитрида ниобия измерялись четырехконтактным методом при температуре $13.5 \mathrm{~K}$. Измерения проводились с помощью криомагнитной системы $8 \mathrm{~T}$ CryoFree-404, позволяющей охлаждать образцы до $6 \mathrm{~K}$ в постоянном магнитном поле с индукцией до 8 Т. Поле было направлено перпендикулярно плоскости образцов. Температура измерялась с помощью датчика Cernox CX-1050 и контроллера LakeShore 335. Погрешность измерения температуры была меньше $0.01 \mathrm{~K}$. Для формирования одиночного импульса через токовые контакты и регистрации отклика с потенциальных контактов использовались генератор АСК-4106 и осциллограф АСК-3107 с частотными полосами пропускания не ниже $100 \mathrm{MHz}$. Подробнее техника измерений и параметры пленок приведены в работе [12].

Для контроля перегрева образцов ток пропускался за разное время $t_{0}$ : от 25 до $250 \mu \mathrm{s}$. При этом ВАХ образцов совпадали в пределах ошибки измерений (вставка на рис. 3). Важно отметить, что если для оценки нагрева пленки применять элементарный расчет без учета теплоотвода в потенциальные контакты и подложку, то получается неверный результат - рост температуры на десятки градусов.

\section{4. Обсуждение}

При моделировании процесса теплопереноса в системе пленка-подложка тепло, отводимое от пленки в потенциальные контакты, учитывалось значением параметра $H$, которое в данном случае определялось в том числе и толщиной пленки. Так как нагреванием контак- 
тов можно пренебречь, то моделирование распределения тепла в контактах является излишним.

Граничное условие при $x=d$ в моделировании не учитывалось, так как коэффициент температуропроводности пленки близок по величине к коэффициенту температуропроводности подложки $\left(a^{2} \approx 4 \cdot 10^{-2} \mathrm{~cm}^{2} / \mathrm{s}\right)$. Уравнение теплопроводности содержит только один параметр - коэффициент температуропроводности.

Правое граничное условие (подложка-медная основа) является довольно „жестким“, потому что медная основа, к которой крепилась подложка с пленкой, практически не нагревается в силу очень высокой теплопроводности меди при температуре эксперимента и массы, превышающей массу подложки на 3-4 порядка.

\section{Заключение}

Таким образом, в работе решено неоднородное уравнение теплопроводности для системы пленка-подложка с граничными условиями, учитывающими теплоотвод от пленки в контакты, подложку и медную основу (термостат). Показано, что применение контактов из бериллиевой бронзы позволяет проводить транспортные измерения с токами высокой плотности $\left(10^{4}-10^{5} \mathrm{~A} / \mathrm{cm}^{2}\right)$ без значительного нагрева образцов.

\section{Конфликт интересов}

Авторы заявляют, что у них нет конфликта интересов.

\section{Список литературы}

[1] F.W. Carter, T. Khaire, C. Chang, V. Novosad. Appl. Phys. Lett., 115 (9), 092602 (2019). https://doi.org/10.1063/1.5115276

[2] Y. Ren, D. Zhang, K. Zhou, W. Miao, W. Zhang, S. Shi, V. Seleznev, I. Pentin, Y. Vakhtomin, K. Smirnov. AIP Advances. 9 (7), 075307 (2019). DOI: 10.1063/1.5090132

[3] Y. Suzuki, N. Iguchi, K. Adachi, A. Ichiki, T. Hioki, C.-W. Hsu, R. Sato, S. Kumagai, M. Sasaki, J.-H. Noh, Y. Sakurahara, K. Okabe, O. Takai, H. Honma, H. Watanabe, H. Sakoda, H. Sasagawa, H. Doy, S. Zhou, H. Hori, S. Nishikawa, T. Nozaki, N. Sugimoto, T. Motohiro. J. Phys.: Conf. Series. 897 (Conf. 1), 012019 (2017). DOI:10.1088/1742-6596/897/1/012019

[4] N. Sugimoto, N. Iguchi, Y. Kusano, T. Fukano, T. Hioki, A. Ichiki, T. Bessho, T. Motohiro. Supercond. Sci. Technol., 30 (1), 015014 (2016). DOI:10.1088/0953-2048/30/1/015014

[5] N. Sugimoto, T. Motohiro. Vacuum, 93, 13 (2013). http://dx.doi.org/10.1016/j.vacuum.2012.12.002

[6] Д.М. Гохфельд. ФТТ, 56 (12), 2298 (2014). https://journals.ioffe.ru/articles/viewPDF/41115 [D.M. Gokhfeld. Phys. Sol. St., 56 (12), 2380 (2014). https://link.springer.com/article/10.1134/S1063783414120129]

[7] Справочник конструктора точных приборов. Под ред. И.Я. Левина. (ОБОРОНГИЗ, М., 1953), с. 552.

[8] Таблицы фбизических величин. Справочник. Под ред. акад. И.К. Кикоина. (Атомиздат, М., 1976), 1008 с.
[9] А. Миснар. Теплопроводность твердых тел, жсидкостей, газов и их композиций (Мир, М., 1968), 464 с.

[10] Л.И. Турчак, П.В. Плотников. Основы численных методов (Физматлит, М., 2005), с. 230.

[11] Б.М. Будак, А.А. Самарский, А.Н. Тихонов. Сборник задач по математической бизики (Наука, М., 1972)

[12] М.А. Васютин, Н.Д. Кузьмичев, Д.А. Шилкин. ФТТ, 58 (2), 231 (2016). https://journals.ioffe.ru/articles/viewPDF/42701 [M.A. Vasyutin, N.D. Kuz'michev, D.A. Shilkin. Phys. Sol. St., 58 (2), 236 (2016). DOI: 10.1134/S1063783416020311] 